\title{
Health Literacy, Vulnerable Patients, and Health Information Technology Use: Where Do We Go from Here?
}

\author{
Courtney R. Lyles, $P h D^{1,2}$ and Urmimala Sarkar, MD, MPH ${ }^{1,2}$ \\ ${ }^{1}$ Center for Vulnerable Populations, University of California-San Francisco, San Francisco, CA, USA; ${ }^{2}$ UCSF Division of General Internal Medicine, San \\ Francisco General Hospital, San Francisco, CA, USA.
}

J Gen Intern Med 30(3):271-2

DOI: $10.1007 / \mathrm{s} 11606-014-3166-5$

(C) Society of General Internal Medicine 2015

T $\mathrm{n}$ the past few years, the rapid adoption of health informa1 tion technology (HIT) in the US has been very apparent, largely driven by the more than $\$ 25$ billion in incentives for "Meaningful Use" of HIT provided to date as a part of healthcare reform in the Health Information Technology for Economic and Clinical Health (HITECH) Act. As we move into Stage 2 of the Meaningful Use program with new metrics for patient engagement, healthcare leaders are now shifting their focus from implementation to understanding patient needs and preferences for health technology use. The article by Levy et al. ${ }^{1}$ in this issue highlights an important issue in this landscape: the intersection of vulnerable populations and HIT use.

At the national level, the Pew Research Center's Internet and American Life Project estimates that the gaps in technology use (that is, the "digital divide") are shrinking. In 2014, $87 \%$ of US adults accessed the Internet including $81 \%$ of African Americans, $83 \%$ of Hispanics/Latinos, and $77 \%$ of those making less than $\$ 30,000$ annually. ${ }^{2}$ Thus, many view HIT as a mechanism to reach diverse populations to engage in health.

Despite this, there is not much evidence about how HIT use will affect health outcomes or address existing health disparities. The underlying assumption behind incentives to encourage health systems to implement patient portals is that these portals will improve health. While the evidence is far from conclusive, studies do suggest that patient portals can improve processes of care and adherence. ${ }^{3,4}$ Technology is also thought to benefit health through improved patient activation and selfmanagement achieved via access to online health information and peer support. ${ }^{5,6}$ However, clear and consistent evidence related to health disparities is lacking.

In this issue of JGIM, Levy et al. have honed in on an important subgroup where the digital divide persists: those aged 65 years or older, only $57 \%$ of whom are Internet users. Within this older group, the authors were able to explore additional predictors of Internet use - namely, health literacy, which has been previously shown to predict online portal use. ${ }^{7}$

Published online January 15, 2015
They also explored patterns of searching for health information online as opposed to simply noting the presence or absence of any Internet use. The results aren't surprising when examining the barriers of age and health literacy simultaneously: only $32 \%$ of older adults used the Internet to get health information and only $10 \%$ of older adults with limited health literacy did so. This was after adjustment for the major potential confounder of cognitive ability.

This pattern is also likely to hold within other subgroups not specifically examined in this study, such as income and race/ethnicity. It is clear from national data that older adults with lower income or from racial/ethnic minority groups are least likely to use the Internet. ${ }^{8}$ For example, only $25 \%$ of low-income older adults use the Internet at all, ${ }^{9}$ compared to more than $50 \%$ of older adults overall. Furthermore, not only are health literacy, income, and race/ethnicity important determinants of Internet use, but these factors are also directly related to disproportionate burden of chronic illness and disparities in health outcomes. This digital divide superimposed on existing disparities may therefore cause vulnerable populations to fall further behind.

As concerning as Levy et al.'s findings are, they likely underestimate the problem of health information technology among vulnerable groups, because they asked patients about searching for health information online rather than using a health system's online patient portal. Searching online for health information using a website like Google or even WedMD might be challenging for older adults with limited health literacy, but using a complex online portal website to manage personal health record data is an even higher bar. Although patient portal usability data is limited, existing studies suggest that these sites are challenging for patients to navigate, especially for those with limited health literacy and numeracy. ${ }^{10,11}$ Therefore, healthcare providers or systems serving predominantly vulnerable patient populations might find it much more difficult to reach Stage 2 Meaningful Use criteria. Missing out on these financial incentives constrains resources further in safety net health systems, potentially exacerbating some of the existing healthcare disparities we see in these patient populations.

So, where do we go from here to address these issues? We see three major areas that need attention. The first is the most basic: access. We cannot forget that certain subpopulations-low-income older adults in particular — still do not go online and may 
need assistance with broadband in their local communities, and with device and Internet affordability. Second, there are large groups who both want and need technology training, especially since large proportions of diverse patient populations report wanting access to electronic access to healthcare information. ${ }^{12}$ This issue of training is not a one-size-fits-all approach: some individuals still need training to be able to use computers or other devices, but others who are already online performing basic searches for healthcare information may need more focused training to be able to perform sophisticated tasks, such as using a secure password. We see the potential for tiered technology training approaches to improve the health literacy status and patient activation levels of vulnerable patients who are interested in accessing technology to help manage their health and healthcare. Such approaches will be most effective if existing community resources, including adult education and public libraries, can be engaged partners in advancing technology and health.

Third, and perhaps most importantly, we have to pay attention to the usability of websites and other technology platforms. If the goal is to leverage technology for patient education and self-management support, then we need to ensure that we send patients to websites that are simple to access, easy to understand, and developed in literacy-appropriate and language-appropriate ways. The true spirit of user-centered design $^{13}$ is to ensure accessibility and functionality across a diverse group of end users, and yet we rarely employ these strategies in creating health technologies. A recent review we conducted found that not only were usability studies of diabetes technologies rarely published, but also that the existing usability studies often did not report on the characteristics of their user populations ${ }^{14}$ - suggesting that limited health literate or low-income groups are almost never included in the design phase. Again, this usability issue might be further amplified with widespread online portal use as a part of Meaningful Use, as the data from integrated systems like Kaiser with longstanding portal access show that racial/ ethnic minorities and those with limited health literacy are less likely to use these websites. ${ }^{7}$

All three of these steps require policy change and investment. From the access and training perspectives, available funds from both federal/state agencies as well as healthcare systems would ensure increased opportunities for more vulnerable patients to be able to use online tools for health management. From the research perspective, we believe that the National Institutes of Health and the Patient-Centered Outcomes Research Institute should fund not only the trials to determine the effectiveness of health technologies, but also play close attention to usability needs of diverse populations and emphasize real-world, implementation science approaches to understand how the tools are used across diverse patient groups. Finally, from a policy perspective, the Office of the National Coordinator can work to directly include health literacy and language in their future Meaningful Use standards for patient engagement with online portal websites. While there are several areas to improve in the coming years, the widespread implementation of health technology nationwide (particularly online portals) offers a more standardized platform for engaging diverse patients in healthcare management across settings. While we believe that the current iteration of patient-facing health information technology leaves much room for improvement, the widespread implementation of portals does increase the reach of technology within American health care systems. With so many hospitals and clinics now using electronic health records and portals, well-designed technology has the potential to make a meaningful population impact.

Acknowledgements: Drs. Lyles and Sarkar are supported by several grants from the Agency for Healthcare Research and Quality (K99022408, R24HSO22047, and P3OHSO23558).

Corresponding Author: Courtney R. Lyles, $\mathrm{PhD}$; Center for Vulnerable PopulationsUniversity of California-San Francisco, San Francisco, CA, USA (e-mail: courtney.lyles@ucsf.edu).

\section{REFERENCES}

1. Levy, et al. J Gen Intern Med. DOI: 10.1007/s11606-014-3069-5

2. Pew Research Internet Project. Internet User Demographics: January 2014. http://www.pewinternet.org/data-trend/internet-use/latest-stats/ Accessed December 1, 2014.

3. Sarkar U, Lyles CR, Parker MM, et al. Use of the refill function through an online patient portal is associated with improved adherence to statins in an integrated health system. Med Care. 2014;52(3):194-201.

4. Tenforde M, Nowacki A, Jain A, Hickner J. The association between personal health record use and diabetes quality measures. J Gen Intern Med. 2012;27(4):420-424.

5. Solomon M, Wagner SL, Goes J. Effects of a Web-based intervention for adults with chronic conditions on patient activation: online randomized controlled trial. J Med Internet Res. 2012;14(1):e32.

6. Ramadas A, Quek KF, Chan CK, Oldenburg B. Web-based interventions for the management of type 2 diabetes mellitus: a systematic review of recent evidence. Int J Med Informat. 2011;80(6):389-405.

7. Sarkar U, Karter AJ, Liu JY, et al. The literacy divide: health literacy and the use of an internet-based patient portal in an integrated health systemresults from the diabetes study of northern California (DISTANCE). J Health Comm. 2010;15(Suppl 2(Journal Article)): 183-196.

8. Pew Research Internet Project. African Americans and Internet Use. http://www.pewinternet.org/2014/01/06/african-americans-and-technology-use/ (Accessed 12/1/14);2014.

9. Pew Research Internet Project. Technology Adoption by Lower Income Populations. http://www.pewinternet.org/2013/10/08/technology-adoption-by-lower-income-populations/ (Accessed 12/1/14);2013.

10. Taha J, Sharit J, Czaja SJ. The impact of numeracy ability and technology skills on older adults' performance of health management tasks using a patient portal. J Appl Gerontol. 2014;33(4):416-436.

11. Segall N, Saville JG, L'Engle P, et al. Usability evaluation of a personal health record. AMIA Annual Symposium Proceedings. 2011;2011:12331242 .

12. Schickedanz A, Huang D, Lopez A, et al. Access, interest, and attitudes toward electronic communication for health care among patients in the medical safety net. J Gen Intern Med. 2013;28(7):914-920.

13. Maguire M. Methods to support human-centered design. Int J HumComput St. 2001;55(4):587-634.

14. Lyles CR, Sarkar U, Osborn CY. Getting a technology-based diabetes intervention ready for prime time: a review of usability testing studies. Curr Diabetes Rep. 2014;14(10):534. 